\title{
Percutaneous Tracheostomy in Coagulopathic Patients: Proceed with Caution
}

\author{
Jigeeshu V Divatia \\ Indian Journal of Critical Care Medicine (2020): 10.5005/jp-journals-10071-23360
}

Percutaneous dilatational tracheostomy (PDT) was first described in 1985. Using the Seldinger technique, the trachea was punctured by a needle, a guidewire was introduced, and the trachea was dilated using serial dilators, following which a tracheostomy tube was inserted over the guidewire. 'The advantages of PDT were quickly recognized and it was adopted enthusiastically by the intensive care community. It could be performed at the bedside by the intensive care team without disruption of the intensive care unit (ICU) routine. No surgical expertise was required, and it reduced the dependence on surgical scheduling for an "ICU tracheostomy." Other PDT techniques were developed, which included the Griggs' guidewiredilator forceps method, ${ }^{2}$ the PercuTwist, ${ }^{3}$ the translaryngeal approach, ${ }^{4}$ a single-stage dilatation using a tapered dilator, ${ }^{5}$ and balloon dilatation of the stoma. ${ }^{6}$ The rate of complications was low, and PDT developed a good track record of safety. ${ }^{7}$ Additional techniques further increased the safety of the procedure: fiberoptic bronchoscopic guidance during needle insertion helped reduce the incidence of posterior tracheal wall puncture, ${ }^{8}$ and ultrasonography (USG) of the neck helped detect the presence of blood vessels in the field, accurately identified tracheal rings, and guided tracheal puncture and guidewire insertion in real time. ${ }^{9-11}$ Coagulopathy was initially considered a contraindication for PDT. Ligation of bleeding vessels and use of electrocautery for hemostasis were difficult to be performed in the ICU environment, and surgical tracheostomy was preferred in patients considered to be at high risk of bleeding. However, with increasing experience, several authors reported safe performance of PDT in patients with coagulopathy. ${ }^{12-15}$ These reports were encouraging, as several critically ill patients have thrombocytopenia, increased international normalized ratio (INR), or activated partial thromboplastin time (aPTT). Patients may be anticoagulated, receiving antiplatelet agents, or drugs for thromboprophylaxis, all of which can increase periprocedural bleeding.

This issue of the Indian Journal of Critical Care Medicine carries two studies that add to the body of literature on the safety of PDT in coagulopathic patients. Praveen Kumar et al. performed a prospective observational study in a gastroenterology and liver transplant ICU at a tertiary care hospital in India. ${ }^{16}$ They enrolled 652 adult patients, of whom 345 (53\%) were coagulopathic, i.e., defined as having a platelet count $\leq 50 \times 10^{9} / \mathrm{L}$, or INR $\geq 1.5$. Percutaneous dilatational tracheostomy was performed under realtime ultrasound guidance. Approximately one third of the patients received fresh frozen plasma (FFP) or platelet transfusions before the procedure. No patient had significant hemorrhage requiring blood transfusion or conversion to surgical tracheostomy. The overall rate of minor bleeding was $3.2 \%$. In another study, ${ }^{17}$ PDT was safely performed in patients with coagulopathy (including refractory coagulopathy) due to liver disease or following liver transplant for acute liver failure.
Department of Anesthesiology, Critical Care and Pain, Tata Memorial Centre, Homi Bhabha National Institute, Mumbai, Maharashtra, India

Corresponding Author: Jigeeshu V Divatia, Department of Anesthesiology, Critical Care and Pain, Tata Memorial Centre, Homi Bhabha National Institute, Mumbai, Maharashtra, India, Phone: +91 9869077435, e-mail: jdivatia@yahoo.com

How to cite this article: Divatia JV. Percutaneous Tracheostomy in Coagulopathic Patients: Proceed with Caution. Indian J Crit Care Med 2020;24(2):85-87.

Source of support: Nil

Conflict of interest: None

In the other study in this issue, Sasane et al. ${ }^{18}$ report a retrospective study of 159 patients who underwent PDT, of whom 87 had a risk factor for bleeding. Of these, $85 \%$ had a coagulopathy, which was defined by the authors as any of thrombocytopenia (platelet count $<100 \times 10^{9} / \mathrm{L}$ ), INR $>1.5$, aPTT $>50$ seconds, or treatment with antiplatelet agents or anticoagulants. The authors did not transfuse FFP or platelets prophylactically and did not interrupt antiplatelet or anticoagulant therapy prior to the procedure. Overall, only two patients had minor bleeding, of which one patient had coagulopathy, which was treated with FFP and platelet transfusion. No patient required red blood cell transfusion or conversion to surgical tracheostomy.

The overall rate of complications was low (5.7\% in both studies). Thus, the conclusion of these studies is that it is safe to perform PDT in patients with coagulopathy. However, these results must be tempered with caution. In both studies, PDT was performed or supervised by experienced operators. A single-stage tapered dilator technique was used and performed after preoperative USG scanning of the neck for vessels in the surgical field and with realtime USG guidance. In Sasane's study, fiberoptic bronchoscopic guidance was used in addition. ${ }^{18}$ Praveen Kumar et al. used a modified technique that notably avoided incising the skin and soft tissue during the procedure. ${ }^{16}$ Thus, both groups of authors took great care to ensure the presence of adequate expertise and equipment for the performance of a safe PDT.

In Praveen Kumar's study, minor bleeding was seen in $4.1 \%$ coagulopathic patients and in $2.3 \%$ patients without coagulopathy. ${ }^{16}$ In the subgroup of patients with significant coagulopathy (platelets $<25 \times 10^{9} /$ L or INR $>2.0$ ), the incidence of bleeding was two times higher than those without significant coagulopathy (5.9\% vs $2.6 \%$, $p=0.10$ ). Similarly, in patients with unsatisfactory neck vascular anatomy and coagulopathy, complications including bleeding occurred in $8.1 \%$ patients vs $4 \%$ patients with satisfactory, good, or excellent neck vascular anatomy with coagulopathy. While statistically significant differences were not observed because of the 
small numbers involved, the occurrence of minor bleeding appears to be numerically higher in patients with coagulopathy than in those without coagulopathy, in patients with severe coagulopathy, and in patients with unfavorable vascular anatomy and coagulopathy.

It appears that aspiration of blood with deterioration of oxygenation, appearance of new pulmonary parenchymal infiltrates, and need for increased inspired oxygen concentration and/or positive end-expiratory pressure was not a significant problem in either study. In Sasane's study, transient hypoxemia occurred in only four patients; it is not clear whether there were differences between coagulopthatic and noncoagulopathic patients. ${ }^{18}$ In Praveen Kumar's study, only three patients $(0.5 \%)$ had desaturation during the procedure and $0.9 \%$ required new onset ventilator support for up to 24 hours after the procedure, with no difference between coagulopathic and noncoagulopathic patients. ${ }^{16}$

These studies do not give any guidance regarding when patients should have their coagulopathy corrected prior to PDT or in whom PDT may be performed without preoperative transfusion of FFP or platelets. Without adequate biological rationale, it may be adventurous to conclude from Sasane's study that "there is no need for interruption of therapy or prior transfusion of platelets or plasma." ${ }^{18}$ In patients with chronic liver disease or major liver resection, the INR may not provide an accurate estimate of the bleeding risk. The INR evaluates only the procoagulant activity and not that of anticoagulants and is therefore not appropriate for the evaluation of the overall procoagulant-anticoagulant balance that may be reset in patients with chronic liver disease ${ }^{19}$ and after major liver resection. ${ }^{20}$ Viscoelastic testing evaluates both pro- and anticoagulant activity and may demonstrate normal, hypercoagulable, or unchanged clot strength in some patients. It is not clear whether these tests were performed in some of the patients in these studies. At our institute, thromboelastography is often performed to help decide whether to correct coagulopathy before PDT or other invasive procedures.

Increasing numbers of patients with severe respiratory failure are now being treated with extracorporeal membrane oxygenation (ECMO). Dimopoulos et al. studied 65 consecutive PDTs performed in patients receiving venovenous (VV) ECMO ${ }^{21}$ In all patients, the periprocedure intravenous heparin protocol was individualized. The majority of patients (43 of 65) had the heparin infusion stopped within 4-12 hours of performing the PDT. Periprocedure coagulation parameters were managed according to the local standards (aPTT $<1.5$, INR $<1.5$, hemoglobin $>7 \mathrm{~g} / \mathrm{dL}$, platelets $>100 \times 109$ cells $/ \mathrm{L}$, and fibrinogen $>1.5 \mathrm{~g} / \mathrm{L}$ ). Seven patients (11\%) had major bleeding, and three of these also required ECMO circuit change due to circuit dysfunction. Two more patients without bleeding required a circuit change. Thus, PDT was associated with a high complication rate in VV-ECMO patients, and complications not only included bleeding in patients but also dysfunction of ECMO circuits.

Thus, PDT in coagulopathic patients is relatively safe but must not be taken lightly. Patients must be made aware of the potential risks and counseled accordingly. Viscoelastic tests of coagulation may help decide whether and which hemostatic factors need to be transfused prior to the procedure. Any decision whether and when to stop or continue therapeutic anticoagulation prior to the PDT must be individualized, taking into account the risks of bleeding vs the risks of thrombosis. Fresh frozen plasma and platelets and factors for transfusion to reverse coagulopathy as well as surgical expertise must be available in short notice, should bleeding occur. Percutaneous dilatational tracheostomy must be performed by experienced operators, with a technique that minimizes the risk of bleeding. The USG scanning of the neck and real-time USG with or without fiberoptic bronchoscopy during the procedure should be used. Thus, PDT can be performed in coagulopathic patients in the ICU, by intensivists with adequate expertise and with abundant caution.

\section{References}

1. Ciaglia P, Firsching R, Syniec C. Elective percutaneous dilatational tracheostomy. A new simple bedside procedure; preliminary report. Chest 1985;87(6):715-719. DOI: 10.1378/chest.87.6.715.

2. Griggs WM, Worthley LI, Gilligan JE, Thomas PD, Myburg JA. A simple percutaneous tracheostomy technique. Surg Gynecol Obstet 1990;170(6):543-545.

3. Frova G, Quintel M. A new simple method for percutaneous tracheostomy: controlled rotating dilation. A preliminary report. Intensive Care Med 2002;28(3):299-303. DOI: 10.1007/s00134-0021218-5.

4. Fantoni A, Ripamonti D. A non-derivative, non-surgical tracheostomy: the translaryngeal method. Intensive Care Med 1997;23(4):386-392. DOI: 10.1007/s001340050345.

5. Byhahn C, Wilke HJ, Halbig S, Lischke V, Westphal K. Percutaneous tracheostomy: Ciaglia blue rhino vs the basic Ciaglia technique of percutaneous dilational tracheostomy. Anesth Analg 2000;91(4): 882-886. DOI: 10.1097/00000539-200010000-00021.

6. Zgoda MA, Berger R. Balloon-facilitated percutaneous dilational tracheostomy tube placement: preliminary report of a novel technique. Chest 2005;128(5):3688-3690. DOI: 10.1378/chest.128.5.3688.

7. Al-Ansari MA, Hijazi MH. Clinical review: percutaneous dilatational tracheostomy. Crit Care 2006;10(1):202. DOI: 10.1186/cc3900.

8. Marelli D, Paul A, Manolidis S, Walsh G, Odim JN, Burdon TA, et al. Endoscopic guided percutaneous tracheostomy:early results of a consecutive trial. J Trauma 1990;30(4):433-435. DOI: 10.1097/00005373-199004000-00012.

9. Rajajee V, Fletcher JJ, Rochlen LR, Jacobs TL. Real-time ultrasoundguided percutaneous dilatational tracheostomy: a feasibility study. Crit Care 2011;15(1):R67. DOI: 10.1186/cc10047.

10. Rudas M, Seppelt I, Herkes R, Hislop R, Rajbhandari D, Weisbrodt L. Traditional landmark vs ultrasound guided tracheal puncture during percutaneous dilatational tracheostomy in adult intensive care patients: a randomised controlled trial. Crit Care 2014;18(5):514. DOI: 10.1186/s13054-014-0514-0.

11. Gobatto AL, Besen BA, Tierno PF. Ultrasound-guided percutaneous dilational tracheostomy vs bronchoscopy-guided percutaneous dilational tracheostomy in critically ill patients (TRACHUS): a randomized non-inferiority controlled trial. Intensive Care Med 2016;42(3):342-351. DOI: 10.1007/s00134-016-4218-6.

12. Kluge $S$, Meyer A, Kühnelt P, Baumann HJ, Kreymann G. Percutaneous tracheostomy is safe in patients with severe thrombocytopenia. Chest 2004;126(2):547-551. DOI: 10.1378/chest.126.2.547.

13. Patel D, Devulapally K, Islam S. Safety of percutaneous tracheostomy in patients with coagulopathy and high ventilatory demand. Chest 2009;136(4):50S. DOI: 10.1016/S0012-3692(16)48047-5.

14. Al Dawood A, Haddad S, Arabi Y, Dabbagh O, Cook DJ. The safety of percutaneous tracheostomy in patients with coagulopathy or thrombocytopenia. Middle East J Anaesthesiol 2007;19(1):37-49.

15. Abouzgheib W, Meena N, Jagtap P, Schorr C, Boujaoude Z, Bartter T. Percutaneous dilational tracheostomy in patients receiving antiplatelet therapy: is it safe? J Bronchology Interv Pulmonol 2013;20(4):322-325. DOI: 10.1097/LBR.0000000000000023.

16. Praveen Kumar G, Govil D, Patel SJ, Jagadeesh KN, Gupta S, Srinivasan $S$, et al. Percutaneous tracheostomy under real-time ultrasound guidance in coagulopathic patients: a single centre experience. Ind J Crit Care Med 2020;24(2).

17. Auzinger G, O'Callaghan GP, Bernal W, Sizer E, Wendon JA. Percutaneous tracheostomy in patients with severe liver disease and 
a high incidence of refractory coagulopathy: a prospective trial. Crit Care 2007;11(5):R110. DOI: 10.1186/cc6143.

18. Sasane SP, Telang MM, Alrais ZF, Alrahma AHNS, Khatib KI. Percutaneous tracheostomy in patients at high risk for bleeding complications - a retrospective single centre experience. Ind J Crit Care Med 2020;24(2).

19. Tripodi A, Mannucci PM. The coagulopathy of chronic liver disease. N Engl J Med 2011;365(2):147-156. DOI: 10.1056/NEJMra1011170.
20. Mallett SV, Sugavanam A, Krzanicki DA, Patel S, Broomhead RH, Davidson BR, et al. Alterations in coagulation following major liver resection. Anaesthesia 2016;71(6):657-668. DOI: 10.1111/anae.13459.

21. Dimopoulos S, Joyce H, Camporota L, Glover G, loannou N, Langrish CJ, et al. Safety of percutaneous dilatational tracheostomy during veno-venous extracorporeal membrane oxygenation support in adults with severe respiratory failure. Crit Care Med 2019;47(2): e81-e88. DOI: 10.1097/CCM.0000000000003515. 